\title{
Medienbildung in der Grundschule - ein konzeptioneller Beitrag zur Auseinandersetzung mit (digitalen) Medien
}

\section{Bardo Herzig}

Zusammenfassung Angesichts der zunehmenden Mediatisierung und Digitalisierung aller Lebensbereiche stellt sich die Frage, wie bereits in der Grundschule Fähigkeiten für ein sachgerechtes, selbstbestimmtes, kreatives und sozial-verantwortliches Handeln unter diesen Bedingungen angebahnt werden können. Ausgehend von einem pädagogischen Medienbegriff, wird ein konzeptioneller Rahmen für die Medienbildung mit Aufgabenfeldern und grundschulspezifischen Kompetenzerwartungen vorgestellt. Dieser sieht eine Auseinandersetzung mit verschiedenen Nutzungsformen (z.B. Information und Lernen oder Spiel und Unterhaltung) und Inhaltsbereichen (z.B. Medienlandschaft und ihre digitale Infrastruktur oder Medieneinflüsse auf das Individuum und die Gesellschaft) vor. Dabei spielen auch informatische Aspekte eine Rolle.

Schlüsselwörter Rahmenkonzept Medienbildung · Medienbegriff · Kompetenzorientierung · Digitalisierung · Informatische Bildung

Prof. Dr. B. Herzig $(\bowtie)$

Institut für Erziehungswissenschaft, Universität Paderborn, Warburger Str. 100, 33098 Paderborn, Deutschland

E-Mail: bardo.herzig@upb.de 


\title{
Media education in primary school - a conceptual framework for dealing with (digital) media
}

\begin{abstract}
In the light of increasing mediatization and digitalization the question suggests itself how to initiate und to develop the ability for appropriate, autonomous, creative and socially responsible acting under these circumstances as early as in primary school. Based on a pedagocical media term, a conceptual framework for media education including primary education specific tasks and competencies is proposed. It allows for dealing with different modes of media use (e.g. information and learning or gaming and entertainment) and content areas (e.g. media landscape and its digital infrastructure or individual and societal impacts of media). At that aspects of computer literacy education are an issue.
\end{abstract}

Keywords Media education framework - Media term · Competence orientation · Digitalization · Computer literacy education

\section{Digitalisierung und Mediatisierung der Lebenswelt}

Mit Beginn der Digitalisierung hat die Entwicklung der Medienlandschaft einen Verlauf genommen, der mit der Entwicklung der (traditionellen) Massenmedien nicht vergleichbar ist (vgl. z. B. Gapski 2016; Breyer-Mayländer 2018; Specht 2018). Mit der Möglichkeit von Computern, analoge Signale in diskrete (binäre) zu wandeln, diese nach mathematischen Regeln zu verarbeiten, die Verarbeitung selbst algorithmisch zu steuern und die Gegenstände und Ergebnisse dieser Verarbeitung in Form von Daten in Netzen zu prozessieren, verändert sich unsere Lebenswelt nachhaltig. Begriffe wie Sensorisierung, Datafizierung, Formalisierung und Algorithmisierung weisen darauf hin, dass immer mehr Zustände, Prozesse und Elemente unserer Lebensumwelt quantifiziert und automatisch verarbeitet werden. Dazu zählen sowohl solche Daten, die wir aktiv selbst zur Verfügung stellen, als auch solche, die im Zuge der Nutzung verschiedener Medien erhoben werden, ohne dass uns dies jeweils bewusst ist. Zunehmend sind aber Algorithmen auch in der Lage, selbst Problemlösungen zu generieren, indem sie aus Beispielen und damit verbundenen großen Datenmengen lernen, z. B. bei der Erkennung von Gesichtern oder der Diagnose von Krankheiten (big data, vgl. Daniel 2019). Die technologischen Entwicklungen haben auch zu Veränderungen der inhaltlichen Medienangebote und ihrer Strukturen geführt. So wachsen Angebote aus dem Bereich des Fernsehens und des Internets zusammen (hybrides Fernsehen) oder Medienangebote werden mit interaktiven Werkzeugen verbunden, z. B. in einem mobilen Endgerät wie dem Smartphone. Dadurch wird zum Teil die Unterscheidung von Medienarten obsolet und die Trennung von Rezeption und Produktion aufgehoben (produser, vgl. Bruns 2018), z. B. im Kontext von sozialen Medien. Die Komplexität der Medienlandschaft und ihre digitale Infrastruktur lassen es aber auch immer schwieriger werden, zwischen dokumentarischen und fiktionalen Beiträgen, zwischen Tatsachenmeldungen und bewusster Irreführung (fake news) und zwischen authentischen Meinungsbeiträgen und durch Roboter erzeugten und verbreiteten Meinungen (social bots) zu unterscheiden (vgl. 
Sachs-Hombach und Zywietz 2018). Zudem ist nicht immer erkennbar, dass und ggf. wie Informationen für den Einzelnen durch algorithmische Verfahren eingeschränkt oder gezielt ausgewählt und zusammengestellt werden (filter bubble) oder wie dadurch das Kauf-, Wahl-, Umwelt- oder Gesundheitsverhalten des Einzelnen beeinflusst wird (persuasive computing, nudging) (vgl. Pariser 2012; Weinmann et al. 2016).

All diese Veränderungen und die damit verbundenen Transformationsprozesse provozieren stärker denn je die Frage, ob und in welcher Form Digitalisierung und Mediatisierung in schulische Erziehungs- und Bildungsprozesse Eingang finden sollten.

\section{Medien in der Schule - Begründungslinien}

Insbesondere im Zusammenhang der technologisch induzierten Entwicklung von Medien und ihrem zunehmenden Einfluss auf alle Lebensbereiche lassen sich verschiedene Begründungslinien für eine schulische Auseinandersetzung mit Medien nachzeichnen (vgl. auch Hawkridge 1990; Herzig 2008; Irion 2018):

- lebensweltbezogene Argumentation: Sie beruht auf der Annahme, dass Kinder und Jugendliche heute in einer medial geprägten (Um-)Welt bzw. Gesellschaft aufwachsen und Schule die Aufgabe zukommt, die gesellschaftlichen Entwicklungen und lebensweltlichen Erfahrungen aufzunehmen und die Heranwachsenden in die Lage zu versetzen, sich in dieser Welt zu orientieren.

- innovations- und produktivitätsbezogene Argumentation: Digitalen Medien kommt eine enorme Bedeutung in gesellschaftlichen Bereichen wie Wissenschaft, Forschung, Handel, Industrie, Dienstleistungssektor, Kultur usw. zu. Schule hat daher die Aufgabe, durch die Auseinandersetzung mit Digitalisierung und Mediatisierung die Grundlagen dafür zu legen, dass die nachfolgende Generation auch zukünftig die Produktivität und Innovationsfähigkeit sicherstellt.

- bildungs- und entwicklungsbezogene Argumentation: In dieser Argumentationsvariante werden insbesondere verkürzte funktionale und instrumentelle Verständnisse von Bildung im Kontext von Digitalisierung und Mediatisierung problematisiert. Schule hat die Aufgabe, eine kritische und (selbst-)reflexive Auseinandersetzung anzuregen und Kompetenzen zu fördern, die Partizipation und aktive Mitgestaltung der digital geprägten Gesellschaft ermöglichen und identitätsstiftende und persönlichkeitsfördernde Wirkung entfalten.

Die medienbezogenen Entwicklungen wurden immer wieder in ordnungspolitischen Dokumenten mit Blick auf schulische Ziele und Aufgaben aufgegriffen. Bereits Anfang der 1980er Jahre legte die Kultusministerkonferenz (KMK) ein Konzept zur Medienerziehung in der Schule vor, gefolgt von einem Gesamtkonzept der BundLänder-Kommission für Bildungsplanung und Forschungsförderung (BLK) zur informationstechnischen (Grund-)Bildung. Der 1995 veröffentlichte Orientierungsrahmen zur Medienpädagogik in der Schule (BLK 1995) sah Medienerziehung als integrative, fächerübergreifende Aufgabe und perspektivisch eine Zusammenführung von Ansätzen zur Leseförderung, Fernseherziehung und informationstechnischen 
Grundbildung vor. In ähnlicher Weise wurde auch in der zeitgleichen Erklärung der Kultusministerkonferenz zur Medienpädagogik in der Schule argumentiert. Gesellschaftliche und kulturelle Teilhabe und die Entwicklung von Medienkompetenz auch im Hinblick auf Digitalisierung, Internet und mobile Endgeräte - formulierte die KMK in ihrer Erklärung zur Medienbildung in der Schule im Jahr 2012 und wollte damit einen Beitrag leisten, „Medienbildung als Pflichtaufgabe schulischer Bildung nachhaltig zu verankern" (KMK 2012, S. 3). Dieser Verpflichtungscharakter wurde mit der Strategie der KMK zur Bildung in der digitalen Welt im Jahr 2016 noch einmal deutlich erhöht, indem sich alle Bundesländer darauf verständigt haben, verpflichtend dafür Sorge zu tragen, dass alle Schülerinnen und Schüler am Ende ihrer Pflichtschulzeit über spezifische Kompetenzen in sechs Bereichen verfügen (vgl. KMK 2016). Dieser Kompetenzrahmen stellt eine wichtige Orientierungshilfe in Bezug auf Anforderungen an eine schulische Medienbildung dar. Allerdings fokussiert er stark auf die Digitalisierung und lässt die damit verbundene Mediatisierung (vgl. Krotz 2017) noch nicht hinreichend zur Geltung kommen. Insofern erscheinen Ergänzungen, Erweiterungen und geeignete Akzentuierungen erforderlich, die auch solche Aspekte einbeziehen, die nicht allein im Zusammenhang von Digitalisierung bedeutsam sind. Dies gilt auch für die Grundschule, die zwar explizit in Bezug auf das „Lernen mit und über digitale Medien und Werkzeuge“ einbezogen ist, weil „Digitalisierung auch außerhalb der Schule alle Lebensbereiche und - in unterschiedlicher Intensität - alle Altersstufen umfasst“" (KMK 2016, S. 11), für die aber keine alters- und entwicklungsspezifischen Kompetenzerwartungen formuliert werden (vgl. auch GV 2015; Irion und Eickelmann 2018, S. 7).

Die hohe Bedeutsamkeit von Digitalisierung in allen Lebensbereichen hat auch den Ruf nach spezifischen informatischen Grundkenntnissen laut werden lassen, die schon im Grundschulalter angebahnt werden sollten.

\section{Informatische Kompetenzen in der Grundschule}

Für die informatische Bildung in der Grundschule hat die Gesellschaft für Informatik (GI) ein Konzept vorgelegt (vgl. GI 2019), in dem explizit betont wird, dass eine Umsetzung der in der KMK-Strategie zur Bildung in der digitalen Welt formulierten Kompetenzen ohne die Informatik nicht möglich sei (vgl. S. VI). Ausgangspunkt einer schulischen Auseinandersetzung sollen informatische Alltagsphänomene sein, die sich in direktem Zusammenhang mit Informatiksystemen - verstanden als Zusammenstellung von Hardware, Software und Vernetzung - zeigen können (z. B. als Smartphone), in indirektem Zusammenhang (z. B. bei einem Ampelsystem) und unabhängig davon (z. B. beim Sortieren von Gegenständen nach bestimmten Kriterien). Neben dem Lebensweltbezug sind Anschaulichkeit, sinnliche Erfahrungsmöglichkeiten, Kompetenzerleben und Selbstwirksamkeit wichtige Grundsätze eines altersund entwicklungsgemäßen Zugangs zu informatischen Fragestellungen. Die konkrete Gestaltung kompetenzorientierter Lernsituationen soll entlang von miteinander verschränkten Prozess- und Inhaltsbereichen geschehen (vgl. S. 2 ff.). Bei den Prozessbereichen geht es zum einen um spezifische informatische Kompetenzen (Modellieren und Implementieren, Strukturieren und Vernetzen), zum anderen um 
eher übergreifende Kompetenzen, die auf informatische Zusammenhänge bezogen werden (Begründen und Bewerten, Kommunizieren und Kooperieren, Darstellen und Interpretieren). Als informatische Gegenstände und Inhalte werden Information und Daten, Algorithmen, Sprachen und Automaten, Informatiksysteme und der Bereich Informatik, Mensch und Gesellschaft genannt. Für die Grundschule werden Kompetenzerwartungen zum Ende der zweiten und der vierten Klasse beschrieben, die als didaktische Empfehlungen verstanden werden und keine empirisch fundierten Stufen darstellen.

Das Verhältnis zwischen Informatik und Medienbildung wird kontrovers diskutiert (vgl. Herzig 2016). Während auf der einen Seite verbindende Elemente betont werden (vgl. z. B. Herzig 2012; GI 2016; Schmid et al. 2018), werden auf der anderen Seite gerade diese integrativen Ansätze mit der Befürchtung verbunden, dass die Bedeutung der Informatik für die Allgemeinbildung nicht erkannt und sie quasi der Medienbildung untergeordnet werde (vgl. Hromkovic 2019). Im vorliegenden Beitrag wird die Auffassung vertreten, dass eine Medienbildung ohne informatische Anteile nicht sinnvoll ist und diese einer professionellen Vermittlung bedürfen, so wie es für andere Fächer auch gilt.

Vor dem Hintergrund der grundsätzlichen Bedeutsamkeit digitaler Medien und der Notwendigkeit, diese auch zum Gegenstand und Mittel schulischen Lehrens und Lernens zu machen, stellt sich die Frage, wie ein Konzept für die schulische Medienbildung strukturiert werden kann und welche grundschulspezifischen Aspekte es dabei zu beachten gilt. Im Folgenden werden entsprechende Überlegungen vom Medienbegriff aus entfaltet.

\section{Medien und Erfahrungsformen}

Im vorliegenden Beitrag wird ein Medienbegriff vertreten, der über die (Erfahrungs-) Formen, in denen wir mit unserer Umwelt in Kontakt treten, entwickelt wird (vgl. Tulodziecki et al. 2019, S. $29 \mathrm{ff}$.). Unterscheiden lassen sich dabei reale Begegnungen mit Sachverhalten oder Personen, modellhafte Formen (z. B. ein Modell einer technischen Anlage), abbildhafte Formen (z.B. Fotos, Filme oder animierte Darstellungen) und symbolische Formen (z.B. gesprochene Sprache oder schriftliche Texte). Da diese Erfahrungsformen in gewisser Weise einen vermittelnden Charakter haben, werden sie manchmal schon selbst als Medien bezeichnet. Aus einer medienpädagogischen Perspektive erscheint es aber zweckmäßig, den Medienbegriff auf technisch vermittelte Erfahrungsformen - und damit auf abbildhafte und symbolische Formen - einzugrenzen. Dies eröffnet in besonderer Weise die Möglichkeit, die Merkmale technisch vermittelter Erfahrungen und Inhalte zu untersuchen und wissenschaftliche Aussagen dazu zu formulieren. Allerdings ist beispielsweise ein Buch als bloßer Gegenstand noch kein Medium, sondern wird erst durch die kommunikativen Zusammenhänge, in denen es steht, zu einem Medium. Dies bedeutet, dass der Medienbegriff sowohl die technischen Geräte bzw. die technologische Basis zur Übertragung, Speicherung, Wiedergabe oder Verarbeitung von abbildhaften und symbolischen Zeichen als auch die dadurch realisierten Medienangebote und deren funktionales Zusammenwirken im Kontext von Kommunikation umfasst (vgl. 


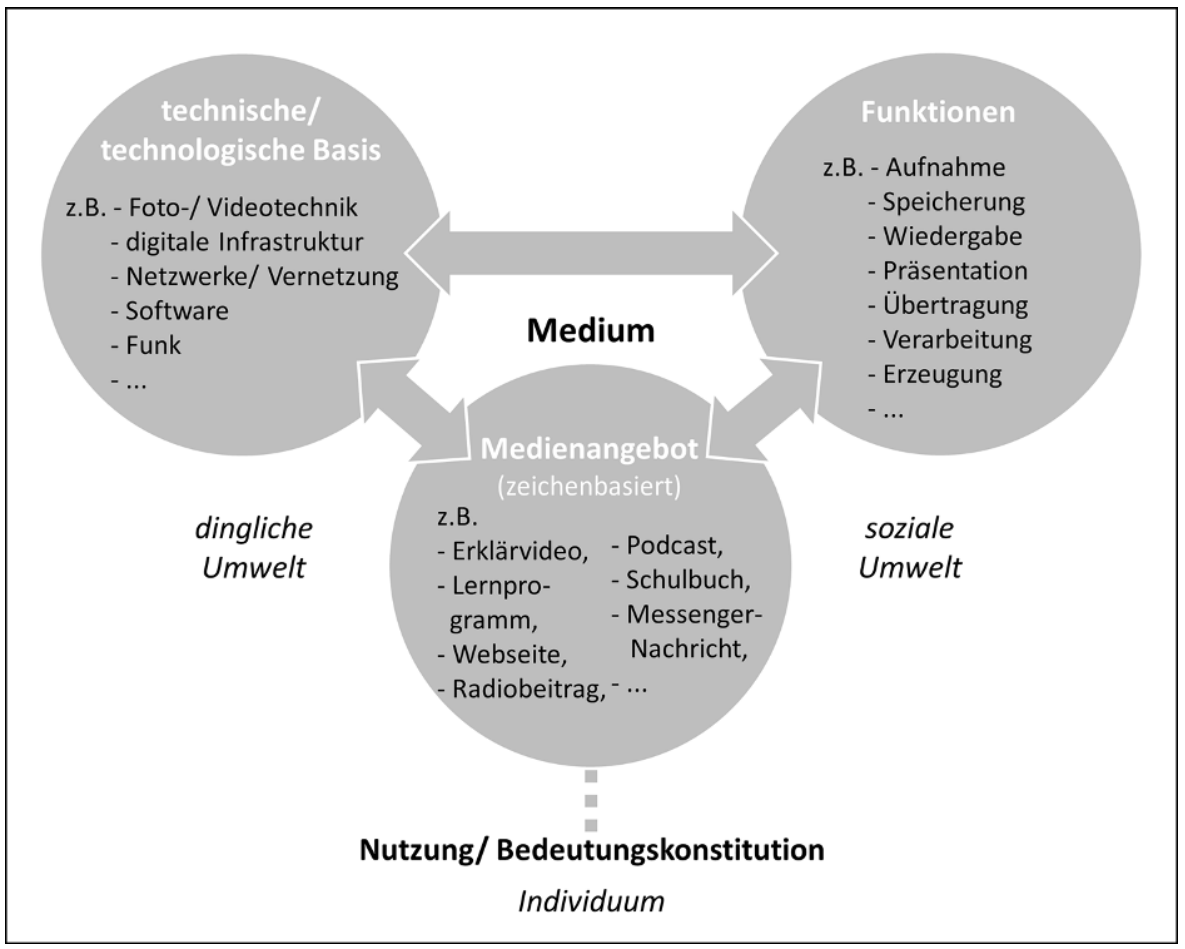

Abb. 1 Darstellungsformen, Funktionen und technische Grundlagen von Medien

Herzig 2012, S. 205 ff.). Medien werden hier somit als Mittler verstanden, durch die in kommunikativen Zusammenhängen (potenzielle) Zeichen mit technischer Unterstützung übertragen, gespeichert, wiedergegeben oder verarbeitet und in abbildhafter oder symbolischer Form präsentiert werden. Der Verweis auf potenzielle Zeichen soll deutlich machen, dass kommunikative Zusammenhänge erst entstehen, wenn die Zeichen als solche von Nutzenden (und Gestaltenden) erkannt und mit Bedeutung versehen werden (vgl. Abb. 1). Die kommunikative Grundkonstellation kann dabei so verstanden werden, dass Medien eine wichtige Rolle im Austausch des Individuums mit seiner sozialen und seiner dinglichen Umwelt und mit sich selbst spielen, z. B. durch Kontakt mit anderen über einen Messenger-Dienst, durch die Rezeption eines Fernsehbeitrags über eine unbekannte Region oder durch ein identitätsstiftendes Profil in einem Netzwerk.

Ein solcher Medienbegriff rekurriert also nicht nur auf das Artefakt als Gegenstand, auf die vermittelnde Funktion und die kommunikative Situation, sondern auch auf die Bedeutung der technischen Bedingtheit dessen, was sich uns als Medienangebot zeichenbasiert darbietet (oder wir selbst als solches gestalten). Auf die besonderen Eigenschaften digitaler Medien wird in der hier vertretenen Definition mit der Funktion der Verarbeitung von Zeichen hingewiesen (zur Präzisierung der Sprechweise der Zeichenverarbeitung vgl. Herzig, 2012, S. 102 ff.). Digitale, d.h. 
computerbasierte, Medien weisen das Alleinstellungsmerkmal auf, Zeichen prozessieren und verarbeiten zu können - sie beruhen auf Rechenmaschinen.

Aus den bisherigen Überlegungen zur Bedeutsamkeit von (digitalen) Medien und zum Medienbegriff lassen sich erste Konsequenzen im Hinblick auf eine schulische Auseinandersetzung formulieren:

- Die starke Ausweitung von digitalen Medien in allen Lebensbereichen sollte Anlass sein, in verschiedenen alltags-, freizeit-, berufs- und gesellschaftsrelevanten Handlungsfeldern Erfahrungen zu sammeln und Kompetenzen zur Orientierung und (Mit-)Gestaltung zu entwickeln.

- Die grundlegende Bedeutung von Zeichen für Medien bzw. Medienangebote sollte dazu führen, ein Verständnis unterschiedlicher Zeichen- und Codierungssysteme anzustreben sowie eine Reflexion über ihre Funktion und Bedeutung bei der auch automatisierten bzw. maschinellen - Produktion von Medienangeboten.

- Die Auseinandersetzung mit der breiten Palette von Medienangeboten auf rezeptiver, produktiver oder interaktiver Ebene sollte nicht nur die wahrnehmbaren medialen Erscheinungsformen umfassen, sondern auch die technischen bzw. technologischen Grundlagen, die ein Verständnis der Phänomene ermöglichen.

- Medien in ihrer Funktion der individuellen und gemeinschaftlichen Klärung des Selbst- und Weltverhältnisses verweisen auf die Bedeutsamkeit, sich in reflexiver Weise mit Einflüssen und Wirkungen von Medien auf verschiedene Lebens- und Gesellschaftsbereiche zu befassen.

Die Frage, welche spezifischen Kenntnisse, Fähigkeiten und Fertigkeiten Kinder und Jugendliche im Medienbereich entwickeln sollten, ist lange Zeit unter dem Leitbegriff der Medienkompetenz diskutiert worden, der in den 1990er-Jahren weitgehend unbestritten war. Mittlerweile hat sich aber - teils in konkurrierender, teils in komplementärer Weise - der Begriff der Medienbildung etabliert (vgl. Tulodziecki et al. 2018).

\section{Medienkompetenz und Medienbildung}

Die Diskussion um Medienkompetenz in Deutschland wurde stark von Baacke beeinflusst, der - ausgehend von kommunikativer Kompetenz im Anschluss an Chomsky und Habermas (vgl. Baacke 1973) - Medienkompetenz als ,Fähigkeit, in die Welt aktiv aneignender Weise auch alle Arten von Medien für das Kommunikations- und Handlungsrepertoire von Menschen einzusetzen“" beschrieb (1996, S. 3). Sie umfasst die Bereiche der Medien-Kritik, der Medien-Kunde, der Medien-Nutzung und der Medien-Gestaltung. In der Folge sind verschiedene Konzepte entstanden, die den Begriff der Medienkompetenz in einem ähnlichen handlungstheoretisch-pädagogischen Verständnis, zum Teil aber auch mit unterschiedlichen Akzentsetzungen und Strukturierungen auslegen (vgl. z. B. Aufenanger 2001; Groeben 2002; Schorb 2005). Gemeinsam ist den Ansätzen, dass der Begriff der Kompetenz in der Regel auf Wissen, Können, Einstellungen und Handlungsmuster von Individuen zielt. In diesem Sinne trägt das hier vertretene Kompetenzverständnis zunächst dem Gedanken Rechnung, dass der Mensch aufgrund seiner Fähigkeit, potentielle Zeichen zu 
deuten und selbst zu erzeugen, in Medienzusammenhängen handeln kann. Insofern kann Medienkompetenz als Vermögen und Bereitschaft des Menschen verstanden werden, in Medienzusammenhängen zu handeln. Mit dem Begriff des „Vermögens“ soll die Annahme ausgedrückt werden, dass die entsprechenden Fähigkeiten mit einem grundsätzlichen Potenzial bzw. den Prädispositionen des Menschen verbunden sind; der Begriff „Bereitschaft“ verweist auf motivationale bzw. volitionale Aspekte von Kompetenz (vgl. Weinert 2001, S. 27 f.). Mit dem Begriff des „Handelns“ ist die Vorstellung eines aktiven Subjekts verbunden, das sein Leben gestalten und seine Fähigkeiten weiterentwickeln kann. Zugleich wird davon ausgegangen, dass Medienkompetenz als Handlungsvermögen und Handlungsbereitschaft der Anregung und Unterstützung bedarf, um zu einer pädagogisch wünschenswerten Ausprägung zu kommen. Dabei ist im Sinne von Zielperspektiven anzustreben, dass die Medienkompetenz soweit entwickelt ist, dass das Individuum bereit und in der Lage ist, auf der Grundlage genereller Kommunikationsfähigkeit in Medienzusammenhängen sowohl sachgerecht und selbstbestimmt als auch kreativ und sozial verantwortlich zu handeln (vgl. KMK 2012, S. 3). Diese Zielperspektiven sind mit der generellen Leitvorstellung verbunden, dass das angestrebte Niveau von Medienkompetenz kulturelle und politische bzw. gesellschaftliche Teilhabe im Sinne eines individuell und gesellschaftlich handlungsfähigen Subjekts ermöglichen soll. Um solche Kenntnisse, Fähigkeiten und Fertigkeiten zu beschreiben, bedarf es einer weiteren Ausdifferenzierung des Konstrukts der Medienkompetenz (s. Abschn. 6).

Der Medienkompetenzbegriff hat von verschiedener Seite Kritik erfahren. Sie bezieht sich z.B. auf eine zu schwache Berücksichtigung von Kritikfähigkeit und reflexiver Haltung gegenüber einer sozialtechnologischen Engführung (vgl. Aufenanger 2000, S. 7), auf die einseitige Betonung von Verfügungswissen gegenüber Orientierungswissen (vgl. Marotzki 2004, S. 64), auf die Konzentration des Verhältnisses von Mensch und Medien gegenüber dem Verhältnis von Mensch und Welt (vgl. Pietraß 2005, S. 44 f.) oder auf die Fokussierung auf Lernvorgänge gegenüber selbstgesteuerten Entwicklungs- und Bildungsprozessen (vgl. Spanhel 2010, S. $51 \mathrm{ff}$.). Eine solche Kritik unterstellt in der Regel ein funktional-pragmatisches Kompetenzverständnis, das insbesondere außerhalb des medienpädagogischen Diskurses dominierte und dem hier vertretenen Kompetenzverständnis gerade nicht zugrunde liegt (s. oben). Insofern besteht kein Anlass, den Kompetenzbegriff durch den Bildungsbegriff zu ersetzen, sondern ihn dazu in Beziehung zu setzen. Eine solche Verbindung kann hergestellt werden, wenn man Medienbildung vornehmlich als Prozessbegriff verwendet und Ansätze zur Medienkompetenz als Grundlage für die Beschreibung wünschenswerter Kompetenzniveaus nutzt. In gewisser Weise stellt der Begriff der Medienbildung damit einen Oberbegriff für alle bildungsrelevanten Aktivitäten mit Medienbezug dar - sowohl in Bezug auf Medien als Mittel und Werkzeug als auch als inhaltlicher Gegenstand von formalen und informellen Bildungsprozessen (vgl. Tulodziecki et al. 2019, S. 41 f., S. 192; vgl. auch Gervé 2019, S. $101 \mathrm{f}$.).

Vor dem Hintergrund eines prozessorientierten Verständnisses von Medienbildung stellt sich insbesondere für die schulische Umsetzung die Frage nach geeigneten Zielvorstellungen und Inhalten als Grundlage für die Planung von Unterrichtseinheiten oder Projekten. Ein wichtiger Schritt dafür ist die Entwicklung eines 
konzeptionellen Rahmens für die Medienbildung, der geeignet erscheint, Themen zu bestimmen, bei deren Behandlung angestrebte Kompetenzen erworben werden können. Zugleich lässt sich ein solcher Rahmen als Grundlage für die Herleitung wichtiger Aufgabenfelder der Medienbildung nutzen.

\section{Konzeptioneller Rahmen zur Medienbildung}

Für einen konzeptionellen Rahmen zur Medienbildung werden im Folgenden verschiedene Dimensionen betrachtet: die Zieldimension, die Dimension medialer Handlungs- und Nutzungsbereiche und die Inhaltsdimension (vgl. Abb. 2). Diese werden zunächst alters- und entwicklungsübergreifend formuliert, bevor dann spezifische Anforderungen an die Grundschule diskutiert und entsprechende Kompetenzerwartungen beschrieben werden.

Mit Bezug auf das skizzierte Kompetenzverständnis und vor dem Hintergrund bestehender Kompetenzmodelle (vgl. z. B. Moser 2006; Tulodziecki 2007; BMBF 2010; Ferrari 2013; LKM 2015) lassen sich folgende Zielbereiche für die Medienbildung nennen:

- Handhabungsfertigkeiten als Basis für eine funktionsgerechte Nutzung von Medien bzw. von Hard- und Software,

- Kommunikationsfähigkeit als Basis für das Verstehen von Medienbotschaften und für eigene mediale Mitteilungen,

- Kenntnisse und Verstehen in verschiedenen Inhaltsbereichen (s. unten) als Grundlage für eine reflexive rezeptive, interaktive und produktive Mediennutzung,

- Recherche- und Strukturierungsfähigkeiten als Voraussetzung zur Nutzung der Medienpotenziale,

\begin{tabular}{|c|c|c|c|c|c|c|}
\hline Zielbereiche: & \multicolumn{6}{|c|}{$\begin{array}{l}\text { Handhabungsfertigkeiten, Kenntnisse, Recherche- und Strukturierungsfähigkeit, Analyse- und Bewertungsfähigkeit, } \\
\text { Kommunikationsfähigkeit, Problemlöse-, Gestaltungs-, Entscheidungs- und Urteilsfähigkeit sowie Handlungstähigkeit } \\
\text { und Handlungsbereitschaft }\end{array}$} \\
\hline \multirow{2}{*}{\multicolumn{2}{|c|}{ Inhaltsbereiche: }} & \multicolumn{5}{|c|}{$\begin{array}{l}\text { Grundformen der Mediennutzung sowie Handlungs- und Nutzungsbereiche: } \\
\text { rezeptive, interaktive und produktive Nutzung von medialen Angeboten oder } \\
\text { Möglichkeiten in den Nutzungs- und Handlungsbereichen }\end{array}$} \\
\hline & & $\begin{array}{l}\text { Information } \\
\text { und Lernen }\end{array}$ & $\begin{array}{l}\text { Analyse und } \\
\text { Simulation }\end{array}$ & $\begin{array}{c}\text { Unterhaltung } \\
\text { und Spiel }\end{array}$ & $\begin{array}{l}\text { Dienst- } \\
\text { leistungen }\end{array}$ & \begin{tabular}{|c|} 
Steuerung \\
und Kontrolle
\end{tabular} \\
\hline \multicolumn{7}{|c|}{$\begin{array}{l}\text { Medienlandschaft und ihre } \\
\text { digitale Infrastruktur }\end{array}$} \\
\hline \multicolumn{7}{|c|}{$\begin{array}{l}\text { Gestaltungsmerkmale und } \\
\text { Erzeugung medialer Botschaften }\end{array}$} \\
\hline \multicolumn{7}{|c|}{$\begin{array}{l}\text { Einfllisse von Medien auf } \\
\text { Individuum und Gesellschaft }\end{array}$} \\
\hline $\begin{array}{l}\text { Bedingungen der I } \\
\text { und Medienverbre }\end{array}$ & - Medienproduktion & & & & & \\
\hline
\end{tabular}

Abb. 2 Konzeptioneller Rahmen zur Medienbildung. (Vgl. Tulodziecki et al. 2019, S. 208) 
- Fähigkeit zur Analyse und Bewertung von Medienangeboten und eigenen Medienbeiträgen,

- Problemlöse-, Entscheidungs-, Gestaltungs- und Urteilsfähigkeit im Medienbereich,

- Handlungsfähigkeit und Handlungsbereitschaft in medialen Kontexten.

Diese Zielbereiche sollten in der schulischen Medienbildung in der Auseinandersetzung mit medialen Handlungs- und Nutzungsbereichen adressiert werden. Sie sind für die Medienbildung bedeutsam, weil die Mediennutzung und das Medienhandeln einerseits selbst bildungsrelevant sein können, z.B. bei der gezielten Verwendung von Medien zum Lernen, und andererseits, weil bildungsrelevante Kenntnisse und Fähigkeiten bei der Mediennutzung und dem Medienhandeln zum Tragen kommen sollen, z. B. wenn die Rezeption einer Nachrichtensendung durch kritische Einschätzungen begleitet wird. Im Folgenden werden - vor dem Hintergrund von rezeptiven, interaktiven und produktiven Nutzungsformen - fünf Handlungs- und Nutzungsbereiche unterschieden:

- Information und Lernen: Die Medienlandschaft bietet vielfältige Angebote, um sich zu informieren und um zu lernen; diese reichen von Werkzeugen der Recherche über Buch-, Radio- und Fernsehbeiträge bis hin zu interaktiven netzbasierten Angeboten.

- Analyse und Simulation: Digitale Medien können genutzt werden, um aus der Analyse von großen Datenmengen neue Erkenntnisse zu erhalten (z. B. durch die Analyse von Patientendaten) oder durch die Simulation von komplexen Prozessen und Systemen Vorhersagen treffen zu können (z. B. Wetterprognosen durch die Simulation von komplexen Modellen).

- Unterhaltung und Spiel: Auch in diesem Bereich finden sich vielfältige Angebote von unterhaltender Literatur über Videoclips und Spielfilme von Streamingdiensten bis hin zu Spielekonsolen und immersiven Spielumgebungen, die mit Hilfe von Datenbrillen erzeugt werden.

- Dienstleistungen: Mit zunehmender Digitalisierung wird auch die Palette von Dienstleistungen im Bereich von Handel, Banken, Haushalt, Verwaltung, Gesundheit und Verkehr breiter, die beispielsweise An- und Verkauf, Beratung oder Information anbieten - teilweise verbunden mit neuen Berufsbildern.

- Steuerung und Kontrolle: Digitale Medien bieten viele Möglichkeiten, Prozesse und Verhalten zu steuern und zu kontrollieren. Dazu zählen sowohl selbst gewählte Möglichkeiten z. B. der Steuerung von Haushaltsfunktionen oder der Überwachung von Körper- und Vitalfunktionen als auch subtile Formen der Beeinflussung z. B. durch personalisierte Werbung oder algorithmisch ausgewählte Informationsangebote.

Über die skizzierten Nutzungs- und Handlungsbereiche sollte auch eine Auseinandersetzung mit spezifischen Inhalten angeregt und ermöglicht werden. Dazu zählen sowohl Inhalte, die aus der medienpädagogischen Diskussion heraus entstanden sind, als auch solche Aspekte, die für ein Verständnis der digitalen Grundlagen der Medienwelt erforderlich sind: 
- Medienlandschaft und ihre digitale Infrastruktur: Dieser Inhaltsbereich umfasst Medienarten und ihre Angebote, Aspekte der Medienkonvergenz, die Überführung von Information in Daten, Datenerfassung (auch durch Sensoren), Modellierung und Algorithmen, Automatisierung und Vernetzung.

- Gestaltungsmerkmale und Erzeugung medialer Botschaften: Für das Verständnis und die Gestaltung von Medienbeiträgen ist es wichtig, sich ihre Zeichenhaftigkeit bewusst zu machen und verschiedene Darstellungsformen und Gestaltungstechniken - insbesondere mit Blick auf das Verhältnis von Form und Inhalt - einer medialen Botschaft zu kennen. Darüber hinaus geht es in diesem Inhaltsbereich um verschiedene Formen der Erzeugung von medialen Botschaften, z.B. durch die Auftragung von Substanzen auf Trägermaterialien (z.B. Briefe oder Bilder), durch technische Einschreibungen in Materialien (z. B. Tonband oder Film) oder durch immaterielle Prozesse mit Hilfe von Algorithmen bzw. Software.

- Medieneinflüsse auf Individuum und Gesellschaft: In diesem Inhaltsbereich geht es um Medieneinflüsse auf Emotionen, Vorstellungen, Verhaltens- und Wertorientierungen sowie auf soziale Zusammenhänge. Thematisiert werden beispielsweise Medien in ihrer Funktion als Stimmungsregulatoren und zum Ausdruck von Emotionen, die Einflüsse von Medien auf die Vorstellungsbildung über Wirklichkeitsbereiche und die verhaltens- und wertbezogenen Wirkungen von Medien, z.B. im Kontext von Gewaltdarstellungen oder exkludierenden und diskriminierenden Beiträgen.

- Bedingungen der Medienproduktion und Medienverbreitung: Technische, rechtliche, ökonomische, personale, institutionelle politische und gesellschaftlich-kulturelle Bedingungen markieren wichtige Voraussetzungen oder Rahmenbedingungen der Produktion und Verbreitung von Medien. Dazu zählen technische Aspekte von Informatiksystemen, Meinungs- und Pressefreiheit, Kinder- und Jugendschutz, Urheberrecht und Datenschutz, Monopolisierung und Datenkapitalismus, Medienkonzerne und ihre Strukturen oder bildungs- und parteipolitische Medienfragen, die in diesem Inhaltsbereich angesprochen werden.

Auf der Grundlage eines solchen Kompetenzrahmens kann eine Vielzahl von Themen für die Medienbildung generiert werden. Angesichts begrenzter Ressourcen ist es wichtig, eine Auswahl an wichtigen Aufgabenfeldern zu bestimmen, in denen schulische Lerngelegenheiten geschaffen werden. Ausgehend vom konzeptionellen Rahmen können die in Abb. 3 genannten stärker nutzungs- bzw. stärker inhaltsbezogenen Aufgabenfelder unterschieden werden.

Für die Aufgabenfelder gilt - wie auch für die Handlungs- und Nutzungsbereiche -, dass sie nicht als trennscharfe oder isolierte Felder zu verstehen sind, sondern sich durchaus überschneiden und bei der Unterrichtsplanung auch bewusst miteinander verbunden werden können.

Vergleicht man die Inhaltsbereiche der GI (vgl. Abschn. 3) mit dem konzeptionellen Rahmen zur Medienbildung, so zeigt sich, dass die dort formulierten Inhaltsbereiche informatische Aspekte explizit integrieren. So werden beispielsweise unter der Inhaltsdimension Medienlandschaft und ihre digitale Infrastruktur die Überführung von Information in Daten und unter Gestaltungsmerkmale und Erzeugung medialer Botschaften verschiedene Darstellungsformen und Möglichkeiten der 


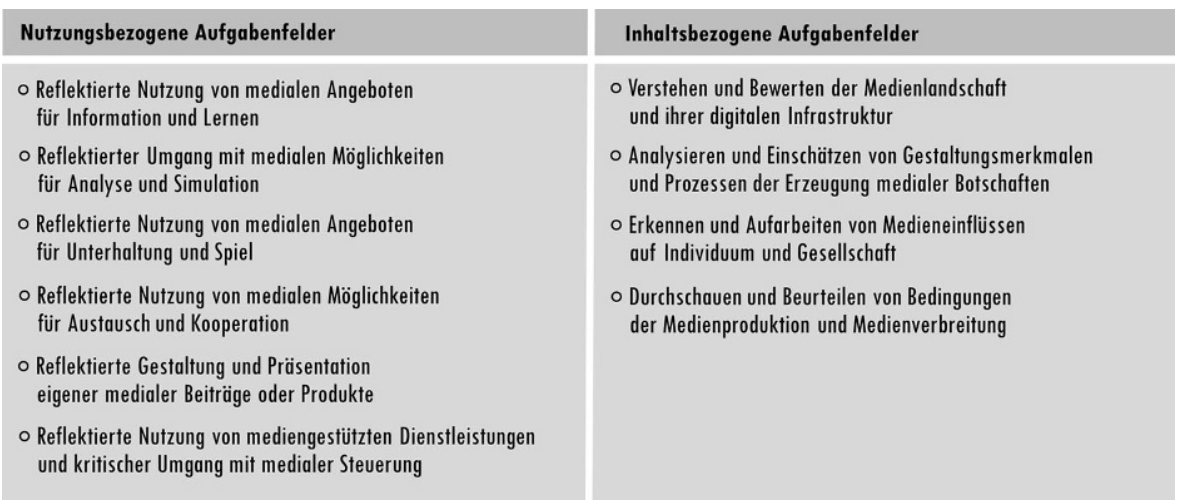

Abb. 3 Aufgabenfelder der Medienbildung

Codierung und Übertragung von Informationen thematisiert (vgl. Tulodziecki et al. 2019, S. 203 f.). Korrespondierend werden im Inhaltsfeld Information und Daten der GI für die Grundschule der Unterschied zwischen Informationen und Daten, die Codierung und Verschlüsselung von Informationen resp. Daten, die Darstellung von Informationen in unterschiedlichen Repräsentationsformen sowie Möglichkeiten der Übermittlung von Informationen thematisiert. Diese Schwerpunktsetzungen (ebenso wie weitere) sind auch unmittelbar mit dem eingangs vorgestellten Medienbegriff verbunden.

Die Kultusministerkonferenz hat in ihrer Strategie zur „Bildung in der digitalen Welt" (erneut) festgeschrieben, dass Medienbildung als integrativer Bestandteil der Fachcurricula zu verstehen sei und innerhalb der bestehenden Unterrichtsfächer und Lernbereiche umgesetzt werden soll. Dazu werden nachfolgend grundschulspezifische Anforderungen in den Blick genommen.

\section{Grundschulspezifische Anforderungen an die Medienbildung}

Die Auseinandersetzung mit Medien im Kindes- bzw. Grundschulalter ist schon im Kontext analoger Medien immer wieder kritisch beleuchtet worden (vgl. Tulodziecki et al. 2019, S. 165 ff.). Diese Kritik setzte sich mit dem Aufkommen digitaler Medien fort und reicht von Befürchtungen in Bezug auf den Verlust von Primärerfahrungen, die Betonung von Wissen gegenüber dem (kritischen) Denken, die Kommerzialisierung von Bildung über Annahmen in Bezug auf problematische Wirkungen durch Reizüberflutung, Gewaltdarstellungen, Suchtverhalten oder Cybermobbing bis hin zu Mahnungen drastischer Auswirkungen auf die Gesundheit (vgl. z. B. Mitzlaff 1996; Frölich und Lehmkuhl 2012). Wenn derartige Problemlagen und mögliche Gefahren der Mediennutzung in bewahrpädagogischen Konsequenzen münden Kinder von problematischen Medieninhalten fernzuhalten und nur mit pädagogisch wertvollen Angeboten zu konfrontieren -, dann wird ein letztlich unmündiges Subjekt unterstellt, das den Medienwirkungen relativ schutzlos ausgeliefert ist. Diese Sichtweise wird in solchen Ansätzen überwunden, die Mediennutzung als einen ak- 
tiven und bedürfnisorientierten Interaktionsprozess auffassen, in dessen Verlauf eine individuelle Bedeutungskonstruktion vor dem Hintergrund sozialer und situativer Kontexte stattfindet. Wenn auch in diesen - handlungs- und entwicklungsorientierten - Ansätzen kein Zweifel daran besteht, dass Kinder bis zu einem gewissen Grad schutzbedürftig sind, so betonen sie doch gleichzeitig das grundsätzliche Ziel von Eigenverantwortlichkeit und Selbstbestimmung.

Grundschulkinder wachsen heute in einer medialen Umwelt auf, die durch ein breites Repertoire an Medien geprägt ist. Neben einer flächendeckenden Ausstattung mit Fernsehen, Smartphone und Internet in den Haushalten, verfügen bereits mehr als die Hälfte der sechs- bis 13-jährigen Kinder über ein eigenes Mobiltelefon, ein Drittel über einen Fernseher und $17 \%$ über einen eigenen Internetzugang (vgl. mpfs 2019, S , 9f.). Am Ende der Grundschulzeit nutzen $81 \%$ der Kinder das Internet mindestens selten, $15 \%$ der 6- bis 7-Jährigen sind bereits täglich online (vgl. ebd., S. $31 \mathrm{f}$.). Mit zunehmendem Alter wird der Anteil regelmäßiger Nutzer für alle Internettätigkeiten größer. Auch digitale Spiele haben für Kinder einen hohen Stellenwert, $42 \%$ der 6- bis 7-Jährigen und 69\% der 10- bis 11-Jährigen nutzen diese täglich oder wöchentlich regelmäßig (vgl. ebd., S. 58). Die Daten zeigen, dass die Grundschulzeit eine wichtige Phase darstellt, in der die Kinder mit unterschiedlichen Medien konfrontiert werden, sich diese aneignen und spezifische Nutzungspraktiken entwickeln. Dieser Prozess wird - abhängig u.a. von sozioökonomischen Faktoren - zwar in unterschiedlicher Weise von Eltern begleitet (vgl. Kammerl et al. 2012), allerdings kann nicht davon ausgegangen werden, dass das Elternhaus allein eine angemessene Grundbildung in diesem Bereich sicherstellen kann. Eine besondere Funktion und Aufgabe kommt hier der Grundschule als erste verpflichtende schulische Einrichtung zu. Sie hat die Aufgabe, allen Kindern Zugänge zur Medienbildung zu ermöglichen, Basiskompetenzen auszubilden, die an vor- und außerschulischen Erfahrungen anknüpfen und Anschlüsse an weiterführende Bildungswege ermöglichen, und im allgemeinbildenden Sinne in ein Grundverständnis der digitalen Welt einführen (vgl. auch Einsiedler 2014). Dabei geht es - vor dem Hintergrund der entwicklungsbezogenen Voraussetzungen in kognitiver, emotionaler und sozial-moralischer Hinsicht - insbesondere auch um die Auslotung des Spannungsfeldes zwischen Kompetenzförderung und Schutzbedürftigkeit.

\section{Grundschulspezifische Kompetenzerwartungen}

Der konzeptionelle Rahmen zur Medienbildung (s. Abschn. 7) bildet eine orientierende Grundlage für die Planung, Abstimmung und Durchführung von Maßnahmen zur schulischen Medienbildung. Für die Grundschule bedeutet dies nicht notwendigerweise, dass alle Handlungs-, Nutzungs- und Inhaltsbereiche vollständig abgedeckt werden müssen. Aufgabe der Grundschule ist es, Grundlagen der Medienbildung zu schaffen, die in den weiterführenden Schulen dann ausgebaut und vertieft werden können. Insbesondere die Handlungsbereiche Analyse und Simulation sowie Dienstleistungen bieten sich für eine Erarbeitung in der Sekundarstufe I an. Insgesamt sollten alle Schülerinnen und Schüler über die Schullaufbahn hin in allen Bereichen Lerngelegenheiten erhalten, um angemessene Kompetenzen entwickeln 
zu können. Entsprechend sollten für einzelne Alters- und Schulstufen Kompetenzerwartungen beschrieben werden, die eine Einschätzung erlauben, welche Kompetenzen wann erworben werden sollten bzw. von welchen Voraussetzungen an einem bestimmten Punkt der Bildungskette ausgegangen werden kann. Differenzierungen der Kompetenzniveaus können anhand von bedürfnis- oder motivationstheoretischen Überlegungen oder mit Bezug auf entwicklungstheoretische Ansätze zur intellektuellen und sozial-moralischen Entwicklung vorgenommen werden (vgl. Herzig und Grafe 2011).

Im Folgenden sind Kompetenzerwartungen für das Ende der Grundschulzeit (Ende 4. Jahrgangsstufe) formuliert (vgl. Tulodziecki et al. 2019, S. 346). Für die Entwicklung dieser Kompetenzen ist insbesondere zu berücksichtigen, dass im Verlauf der Grundschulzeit auch der - für die Mediennutzung zentrale - Schriftspracherwerb stattfindet.

In den Aufgabenfeldern Reflektierte Nutzung von medialen Angeboten für Information und Lernen, Unterhaltung und Spiel sowie Austausch und Kooperation sollte eine reflektierte Nutzung medialer Angebote in folgender Weise angestrebt werden:

- Einzelne mediale und nicht-mediale Möglichkeiten für Information und Lernen

- unter Beachtung von Unterschieden beschreiben,

- im Hinblick auf einzelne Situationen begründet auswählen,

- sachgemäß handhaben und nutzen.

Für den Bereich der Information könnte dies im Einzelnen beispielsweise bedeuten, dass die Schülerinnen und Schüler verschiedene Informationsquellen kennen und anhand von konkreten Merkmalen beschreiben können, für ein bestimmtes Anliegen eine Suchmaschine auswählen, eine konkrete Suchstrategie anwenden und die Ergebnisse im Hinblick auf grundlegende Unterschiede, z. B. Nachricht oder Werbung, einschätzen können.

Im Bereich Austausch könnten sich angemessene Kompetenzen in der Kenntnis verschiedener Möglichkeiten der medienunterstützten Kommunikation (z. B. E-Mail, Messaging-Dienste, Chat, Blogs) zeigen, in der Beschreibung von Unterschieden und möglichen Anwendungssituationen oder in der Kenntnis und Beachtung von Regeln des medienunterstützten Umgangs miteinander.

Im Hinblick auf die Reflektierte Gestaltung und Präsentation eigener medialer Beiträge oder Produkte sollten folgende Kompetenzen entwickelt werden:

- Technische Hilfsmittel für die eigene Gestaltung und Präsentation von Bildern bzw. Fotos, schriftlichen Texten, Hör- und Videobeiträgen beschreiben und funktionsgerecht handhaben.

- Einen Plan für die eigene Gestaltung und Präsentation von Bildern bzw. Fotos, eines Printmediums, eines Hör- oder Videobeitrages unter Anleitung situationsbezogen entwickeln und ausführen.

In Bezug auf einen Videobeitrag würde dies bedeuten, dass die Kinder in der Lage sind, unter Anleitung das Vorgehen zur Erstellung eines Erklärvideos zu einem fachlichen Gegenstand zu beschreiben und die einzelnen Schritte unter Nutzung verschiedener Gestaltungstechniken und eines Tablets umzusetzen. 
In den inhaltsbezogenen Aufgabenfeldern sind folgende Kompetenzen am Ende der Grundschulzeit wünschenswert:

- Verstehen und Bewerten der Medienlandschaft und ihrer digitalen Infrastruktur:

- Ausgewählte Medienarten sowie einzelne Unterschiede und Gemeinsamkeiten zwischen ihnen beschreiben. Beispiele für Medienkonvergenz nennen.

- An Beispielen einzelne bzw. ausgewählte Merkmale der digitalen Infrastruktur beschreiben, z. B. Überführung von Information in Daten, Varianten der Datenerfassung, algorithmisches Vorgehen und Vernetzung.

- Analysieren und Einschätzen von Gestaltungsmerkmalen und Prozessen der Erzeugung medialer Botschaften:

- Ausgewählte Darstellungsformen und Gestaltungstechniken unter Beachtung von Unterschieden beschreiben.

- Bei vorhandenen Medienangeboten und eigenen Medienbeiträgen Darstellungsformen und Gestaltungstechniken bedenken.

- Erkennen und Aufarbeiten von Medieneinflüssen auf Individuum und Gesellschaft:

- Mit Bezug auf Beispiele Gefühle und Realitätsvorstellungen beschreiben, die bei der Mediennutzung hervorgerufen werden können.

- Anhand von Beispielen erläutern, was man gegen unangenehme medienbedingte Gefühle und irreführende Vorstellungen über die Wirklichkeit tun kann.

- Durchschauen und Beurteilen von Bedingungen der Medienproduktion und Medienverbreitung:

- Für ausgewählte Produkte und Leistungen im Medienbereich beschreiben, welche Kosten mit ihnen verbunden sind, und bei der Mediennutzung beachten.

- Bedeutung der Altersbeschränkungen bei Medienangeboten und rechtlicher Bedingungen der Nutzung erläutern können und sie beachten.

Diese Kompetenzerwartungen sind auf einem mittleren Abstraktionsniveau beschrieben und müssen in der Planung von Unterrichtseinheiten weiter konkretisiert werden, z. B. im Hinblick auf Medienarten, Darstellungsformen oder Beispiele einzelner Medienangebote. Im Hinblick auf die schulische Umsetzung bedeutet ein fachintegratives Vorgehen, dass im Rahmen eines Schulentwicklungsprozesses eine Verständigung zwischen Fächern und Lernbereichen erfolgen muss, um über die Grundschullaufbahn abgestimmte Lerngelegenheiten zum Kompetenzerwerb zu schaffen. Anschlusspunkte ergeben sich z.B. an die im Perspektivrahmen Sachunterricht formulierten Kompetenzerwartungen im perspektivenvernetzenden Bereich Medien. Diese sind ebenfalls auf einzelne Handlungs- und Nutzungsbereiche (Informieren, Kommunizieren, Kooperieren, Üben, Spielen, Experimentieren, Gestalten, Präsentieren) bezogen und zielen auf grundlegende Kenntnisse, Nutzungs- und Reflexionsfähigkeiten in den Bereichen des Medienalltags, der Auswirkungen von Medien auf Gesellschaft und Politik, die Sicherheit und der technischen Funktionsweisen (vgl. GDSU 2013, S. 83 ff.).

Funding Open Access funding provided by Projekt DEAL.

Open Access Dieser Artikel wird unter der Creative Commons Namensnennung 4.0 International Lizenz veröffentlicht, welche die Nutzung, Vervielfältigung, Bearbeitung, Verbreitung und Wiedergabe in 
jeglichem Medium und Format erlaubt, sofern Sie den/die ursprünglichen Autor(en) und die Quelle ordnungsgemäß nennen, einen Link zur Creative Commons Lizenz beifügen und angeben, ob Änderungen vorgenommen wurden.

Die in diesem Artikel enthaltenen Bilder und sonstiges Drittmaterial unterliegen ebenfalls der genannten Creative Commons Lizenz, sofern sich aus der Abbildungslegende nichts anderes ergibt. Sofern das betreffende Material nicht unter der genannten Creative Commons Lizenz steht und die betreffende Handlung nicht nach gesetzlichen Vorschriften erlaubt ist, ist für die oben aufgeführten Weiterverwendungen des Materials die Einwilligung des jeweiligen Rechteinhabers einzuholen.

Weitere Details zur Lizenz entnehmen Sie bitte der Lizenzinformation auf http://creativecommons.org/ licenses/by/4.0/deed.de.

\section{Literatur}

Aufenanger, S. (2000). Medien-Visionen und die Zukunft der Medienpädagogik. medien praktisch, 24(93), 4-8.

Aufenanger, S. (2001). Multimedia und Medienkompetenz - Forderungen an das Bildungssystem. In S. Aufenanger, R. Schulz-Zander \& D. Spanhel (Hrsg.), Jahrbuch Medienpädagogik 1 (S. 109-122). Opladen: Leske + Budrich.

Baacke, D. (1973). Kommunikation und Kompetenz. Grundlegung einer Didaktik der Kommunikation und ihrer Medien. München: Juventa.

Baacke, D. (1996). Medienkompetenz als Netzwerk. Reichweite und Fokussierung eines Begriffs, der Konjunktur hat. medien praktisch, 20(78), 4-10.

Breyer-Mayländer, T. (Hrsg.). (2018). Das Streben nach Autonomie: Reflexionen zum digitalen Wandel. Baden-Baden: Nomos.

Bruns, A. (2018). Blogs, Wikipedia, Second Life, and beyond: from production to produsage. New York: Peter Lang.

Bund-Länder-Kommission für Bildungsplanung und Forschungsförderung (1995). Medienerziehung in der Schule. Orientierungsrahmen. Bonn: BLK.

Bundesministerium für Wissenschaft und Forschung (2010). Kompetenzen in einer digital geprägten Kultur. Berlin: BMBF.

Daniel, B. K. (2019). Big data and data science: a critical review of issues for educational research. British Journal of Educational Technology, 50(1), 101-113.

Einsiedler, W. (2014). Grundlegende Bildung. In W. Einsiedler, M. Götz, A. Hartinger, F. Heinzel, J. Kahlert \& U. Sandfuchs (Hrsg.), Handbuch Grundschulpädagogik und Grundschuldidaktik (S. 225-233). Bad Heilbrunn: Klinkhardt/ UTB.

Ferrari, A. (2013). DIGCOMP: A Framework for Developing and Understanding Digital Competence in Europe. JRC Scientific and Policy Reports. European Commission. https://www.rebiun.org/sites/ default/files/2017-11/JRC83167.pdf. Zugegriffen: 5. Juni 2019.

Frölich, J., \& Lehmkuhl, G. (2012). Computer und Internet erobern die Kindheit. Stuttgart: Schattauer.

Gapski, H. (2016). Medienkompetenz 4.0? Entgrenzungen, Verschiebungen und Überforderungen eines Schlüsselbegriffs. merz medien + erziehung, 60(4), 19-25.

GDSU [Gesellschaft für Didaktik des Sachunterrichts] (Hrsg.). (2013). Perspektivrahmen Sachunterricht. Bad Heilbrunn: Klinkhardt.

Gervé, F. (2019). Digitalisierung und Bildung im Primarbereich. In J. Heider-Lang \& A. Merkert (Hrsg.), Digitale Transformation in der Bildungslandschaft - den analogen Stecker ziehen? (S. 98-114). Augsburg: Rainer Hampp.

GI [Gesellschaft für Informatik e.V] (Hrsg.). (2016). Dagstuhl-Erklärung. Bildung in der digitalen vernetzten Welt. Eine gemeinsame Erklärung der Teilnehmerinnen und Teilnehmer des Seminars auf Schloss Dagstuhl - Leibniz-Zentrum für Informatik GmbH. https://gi.de/fileadmin/GI/Hauptseite/Themen/ Dagstuhl-Erkla_rung_2016-03-23.pdf. Zugegriffen: 5. Juni 2019.

GI [Gesellschaft für Informatik e.V] (2019). Kompetenzen für informatische Bildung im Primarbereich. Beilage zu LOG IN, 39(191/192). http://dl.gi.de/handle/20.500.12116/20121. Zugegriffen: 9. Januar 2020.

Groeben, N. (2002). Dimensionen von Medienkompetenz: Deskriptive und normative Aspekte. In N. Groeben \& B. Hurrelmann (Hrsg.), Medienkompetenz. Voraussetzungen, Dimensionen, Funktionen (S. 160-197). München: Juventa. 
GV [Grundschulverband] (2015). Standpunkt Medienbildung. Rundschulkinder bei der Mediennutzung begleiten und innovative Lernpotenziale in der Grundschule nutzen. https://grundschulverband.de/ wp-content/uploads/2017/02/medienbildung.pdf. Zugegriffen: 5. Juni 2019.

Hawkridge, D. (1990). Who needs computers in schools, and why? Computers and Education, 15(1-3), $1-6$.

Herzig, B. (2008). Schule und digitale Medien. In U. Sander, F. v. Gross \& K.-U. Hugger (Hrsg.), Handbuch Medienpädagogik (S. 498-504). Wiesbaden: VS.

Herzig, B. (2012). Medienbildung. Grundlagen und Anwendungen. Handbuch Medienpädagogik, Bd. I. München: kopaed.

Herzig, B. (2016). Medienbildung und Informatische Bildung - Interdisziplinäre Spurensuche. MedienPädagogik. Zeitschrift für Theorie und Praxis der Medienbildung, 25, 59-79.

Herzig, B., \& Grafe, S. (2011). Medienkompetenz - Grundbegriffe, Kompetenzmodelle, Standards. In K. Eilerts, A. H. Hilligus, G. Kaiser \& P. Bender (Hrsg.), Kompetenzorientierung in Schule und Lehrerbildung. Perspektiven der bildungspolitischen Diskussion, der Bildungsforschung und der Mathematik-Didaktik (S. 87-108). Münster: LIT.

Hromkovic, J. (2019). Informatik im Kontext der allgemeinen Bildung. Informatik Spektrum, 42(2), 80-87.

Irion, T. (2018). Wozu digitale Medien in der Grundschule? Sollte das Thema Digitalisierung in der Grundschule tabuisiert werden? Grundschule aktuell, 142, 3-7.

Irion, T., \& Eickelmann, B. (2018). Digitale Bildung in der Grundschule: 7 Handlungsansätze. Grundschule, 50(7), 7-12.

Kammerl, R., Hirschhäuser, L., Rosenkranz, M., Schwinge, C., Hein, S., Wartberg, L., \& Petersen, K.U. (Hrsg.). (2012). EXIF - Exzessive Internetnutzung in Familien. Zusammenhänge zwischen der exzessiven Computer- und Internetnutzung Jugendlicher und dem (medien-)erzieherischen Handeln in den Familien. Lengerich: Pabst Science Publishers.

KMK [Kultusministerkonferenz] (2012). Medienbildung in der Schule. Beschluss der Kultusministerkonferenz vom 08.03.2012. Bonn: Sekretariat der KMK.

KMK [Kultusministerkonferenz] (2016). Bildung in der digitalen Welt. Strategie der Kultusministerkonferenz. Beschluss vom 08.12.2016. Berlin: Sekretariat der KMK.

Krotz, F. (2017). Sozialisation in mediatisierten Welten. Mediensozialisation in der Perspektive des Mediatisierungsansatzes. In I. D. Hoffmann, F. Krotz \& W. Reißmann (Hrsg.), Mediatisierung und Mediensozialisation. Prozesse - Räume - Praktiken (S. 21-40). Wiesbaden: Springer VS.

LKM [Länderkonferenz MedienBildung] (2015). Kompetenzorientiertes Konzept für die schulische Medienbildung. Positionspapier. Stand 29.01.2015. https://lkm.lernnetz.de/files/Dateien_lkm/Dokumen te/LKM-Positionspapier_2015.pdf. Zugegriffen: 5. Juni 2019.

Marotzki, W. (2004). Von der Medienkompetenz zur Medienbildung. In R. Brödel \& J. Kreimeyer (Hrsg.), Lebensbegleitendes Lernen als Kompetenzentwicklung. Analysen - Konzeptionen - Handlungsfelder (S. 63-74). Bielefeld: wbw Bertelsmann.

Mitzlaff, H. (1996). „Computer“ - ein Thema für die Grundschulpädagogik ? Vom Tabu zur Erfahrung im alltäglichen Unterricht. In H. Mitzlaff (Hrsg.), Handbuch Grundschule und Computer (S. 19-49). Weinheim: Beltz.

Moser, H. (2006). Standards für die Medienbildung. Computer + Unterricht, 16(63), 16-18; 49-55.

mpfs [Medienpädagogischer Forschungsverbund Südwest] (2019). KIM-Studie 2018. Kindheit, Internet, Medien. Basisuntersuchung zum Medienumgang 6- bis 13-Jähriger in Deutschland. Stuttgart: mpfs.

Pariser, E. (2012). Filter Bubble: wie wir im Internet entmündigt werden. München: Hanser.

Pietraß, M. (2005). Für alle alles Wissen jederzeit. In H. Kleber (Hrsg.), Perspektiven der Medienpädagogik in Wissenschaft und Praxis (S. 39-50). München: kopaed.

Sachs-Hombach, K., \& Zywietz, B. (2018). Fake News, Hashtags \& Social Bots: Neue Methoden populistischer. Propaganda. Wiesbaden: Springer VS.

Schmid, U., Weitz, K., \& Gärtig-Daugs, A. (2018). Informatik in der Grundschule. Informatik Spektrum, 3(41), 200-207.

Schorb, B. (2005). Medienkompetenz. In B. Schorb, A. Hartung-Griemberg \& C. Dallmann (Hrsg.), Grundbegriffe der Medienpädagogik (S. 254-262). Müchen: kopaed.

Spanhel, D. (2010). Medienbildung statt Medienkompetenz? merz medien + erziehung, 54(1), 49-54.

Specht, P. (2018). Die 50 wichtigsten Themen der Digitalisierung: Künstliche Intelligenz, Blockchain, Bitcoin, Virtual Reality und vieles mehr verständlich erklärt. München: Redline.

Tulodziecki, G. (2007). Was Schülerinnen und Schüler im Medienbereich wissen und können sollen Kompetenzmodell und Bildungsstandards für die Medienbildung. medienImpulse, 15(59), 24-35.

Tulodziecki, G., Herzig, B., \& Aßmann, S. (2018). Der Diskurs um Medienkompetenz und Medienbildung und seine Bedeutung für die Theorieentwicklung in der Medienpädagogik. In I. M. Lüders \& W. Me- 
seth (Hrsg.), Theorieentwicklungen in der Erziehungswissenschaft. Befunde - Problemanzeigen Desiderata (S. 37-50). Bad Heilsbrunn: Klinkhardt.

Tulodziecki, G., Herzig, B., \& Grafe, S. (2019). Medienbildung in Schule und Unterricht. Bad Heilbrunn: Klinkhardt / UTB.

Weinert, F.E. (2001). Vergleichende Leistungsmessung in Schulen - eine umstrittene Selbstverständlichkeit. In F. E. Weinert (Hrsg.), Leistungsmessungen in den Schulen (S. 17-31). Weinheim: Beltz.

Weinmann, M., Schneider, C., \& vom Brocke, J. (2016). Digital nudging. Bus Inf Syst Eng, 58(6), 433-436. 